\title{
666 DELETION OF DRP1 IN T CELLS INCREASES OXPHOS AND CD8+ MEMORY T CELL POPULATION
}

Timothy Bullock, Marissa Gonzales*. University of Virginia, Charlottesville, VA, United States

Background Memory CD8 $\mathrm{T}$ cells are able to more rapidly respond to a secondary exposure to viruses or tumor antigens than naïve $\mathrm{T}$ cells. These memory cells have been shown in vitro to exhibit more elongated mitochondria, more mitochondrial mass, and high spare respiratory capacity, allowing for a rapid response time. ${ }^{12}$ During the activation and differentiation of $\mathrm{T}$ cells, the metabolic demands fluctuate between the necessity for glycolysis or oxidative phosphorylation (OXPHOS), which is supported by shifting of the mitochondrial network state.

Methods Mice are given aCD40, PolyI:C, and OVA protein IP. Cells stimulated in vitro are treated with $5 \mathrm{mg} / \mathrm{mL}$ aCD3, 2 $\mu \mathrm{g} / \mathrm{mL} \mathrm{aCD} 28$, and 10 IU IL2.

Results We hypothesized that manipulating $\mathrm{T}$ cells to elongate mitochondria would provide a metabolic benefit to effector functions and could ultimately increase the function of tumor infiltrating lymphocytes. Using mice lacking Drp1, a mitochondrial fission protein, in $\mathrm{T}$ cells as a model of elongated mitochondria, we see increased spare respiratory capacity and OXPHOS as compared to WT T cells with fission capability. Interestingly, we find that these mice are more likely to generate memory precursor $\mathrm{CD} 8 \mathrm{~T}$ cells as represented by KLRG1lo and CD127hi during a primary response to aCD40, PolyI:C, and ovalbumin protein. As indicated by the increase in memory precursors, we find that Drp1-/- T cells form more CD8 + memory than WT animals after a 28 day rest period. Mice were then challenged with an adenovirus expressing ovalbumin to elicit a recall response. This recall response of CD8 $\mathrm{T}$ cells is greater in animals lacking Drp1 in the T cells. Additionally, $\mathrm{T}$ cells treated with pharmacological reagents M1 and Mdivi to inhibit mitochondrial fission and induce fusion show increased spare respiratory capacity. We are currently testing if this pharmacological method and others can increase spare respiratory capacity in tumor infiltrating lymphocytes. Further studies of differentiation and contraction are still required to determine how the memory $\mathrm{CD} 8 \mathrm{~T}$ cell population is increased. In addition to the increase in $\mathrm{T}$ cell numbers seen, we anticipate an increase in metabolic function. The mouse model here has Drp1-/- CD4 T cells and we are determining if the CD8 $\mathrm{T}$ cell effects are intrinsic by adoptively transferring memory CD8 T cells to WT mice as well as creating mixed chimera mice. We ultimately aim to exploit control of mitochondria to increase memory $\mathrm{T}$ cell development and metabolism as well as control of solid tumors.

Conclusions N/A

\section{REFERENCES}

1. van der Windt GJ, Everts B, Chang CH, Curtis JD, Freitas TC, Amiel E, Pearce EJ, Pearce EL. Mitochondrial respiratory capacity is a critical regulator of CD8+ T cell memory development. Immunity 2012;36(1):68-78.

2. van der Windt GJ, O'Sullivan D, Everts B, Huang SC, Buck MD, Curtis JD, Chang $\mathrm{CH}$, Smith AM, Ai T, Faubert B, Jones RG, Pearce EJ, Pearce EL. CD8 memory T cells have a bioenergetic advantage that underlies their rapid recall ability. Proc Natl Acad Sci U S A. 2013;110(35):14336-41.

http://dx.doi.org/10.1136/jitc-2021-SITC2021.666 